\section{PSICOLOGIA IBEROAMERICANA}

\section{Psicología lberoamericana}

ISSN: 1405-0943

psicología.iberoamericana@uia.mx

Universidad Iberoamericana, Ciudad de

México

México

Moysén Chimal, Alejandra; Garay López, Julieta; Gurrola Peña, Margarita; Esteban Valdés, Juana María de la Luz; Balcázar Nava, Patricia Acontecimientos productores del estrés y manejo en pacientes con cáncer Psicología Iberoamericana, vol. 20, núm. 1, enero-junio, 2012, pp. 63-70

Universidad Iberoamericana, Ciudad de México

Distrito Federal, México

Disponible en: http://www.redalyc.org/articulo.oa?id=133924623007

- Cómo citar el artículo

- Número completo

- Más información del artículo

- Página de la revista en redalyc.org

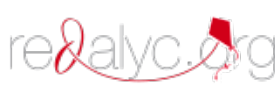

Sistema de Información Científica

Red de Revistas Científicas de América Latina, el Caribe, España y Portugal

Proyecto académico sin fines de lucro, desarrollado bajo la iniciativa de acceso abierto 


\title{
Acontecimientos productores del estrés y manejo en pacientes con cáncer
}

\author{
Events that Produce Stress and Handling Stress in Patients with Cancer
}

\author{
Alejandra Moysén Chimal \\ Julieta Garay López \\ Margarita Gurrola Peña \\ Juana María de la Luz Esteban Valdés \\ Patricia Balcázar Nava* \\ Facultad de Ciencias de la Conducta \\ Universidad Autónoma del Estado de México, México
}

\section{RESUMEN}

El estrés psicosocial es una relación particular entre el individuo y el entorno, que es evaluado como amenazante o desbordante de sus recursos y que pone en peligro su bienestar (Lazarus \& Folkman, 1984). Estos autores también mencionan que su manejo depende de los recursos con los que cuenten las personas. En el caso de los pacientes con alguna enfermedad crónica Taylor (2007) refiere que los recursos de afrontamiento pueden ser confrontativos o evitativos. Se trabajó con 84 personas diagnosticadas con cáncer y que acuden a consulta a un centro oncológico del estado de México, se aplicó el Instrumento de Acontecimientos Productores del Estrés de Caballo y el de estrategias de afrontamiento. De acuerdo con los resultados obtenidos los acontecimientos de agresión física o ambiental correlacionan positivamente con la estrategia de afrontamiento de planeación y afrontamiento activo, aceptación y evasión. Los acontecimientos de muerte o problemas de salud correlacionan positivamente con planeación y afrontamiento activo, apoyo social, aceptación y evasión.

Descriptores: acontecimientos productores del estrés, afrontamiento, cáncer.

\section{ABSTRACT}

Psychosocial stress is a particular relationship between the individual and the environment, which is assessed as threatening or overwhelming their resources and endangering their well-being (Lazarus \& Folkman, 1984); these authors also mentioned that the management of it, depends on the resources available to people. In the case of patients with chronic illness Taylor (2007) refers to the coping of resources may be confrontational or avoided. The research was performed with 84 people diagnosed with cancer patients in an oncology center in the State of Mexico, the instrument applied was Stress Producing Events from Caballo and Strategic Coping. According to the results events of physical or environmental aggression correlate positive with the strategy of coping planning and coping active, acceptance and avoidance. Events of death or health problems correlate positive with planning and active coping, social support, acceptance and avoidance.

Keywords: stress producing events, coping, cancer.

\footnotetext{
*Para correspondencia: Dra. Alejandra Moysén Chimal. Dirección: José Manuel Herrera 136, colonia Morelos, Toluca, Estado de México. C. P. 50120, tel. 7222440300, e-mail: amoysen@hotmail.com. Dra. Julieta Garay López. Dirección: Paseo San Jorge 221, fraccionamiento San Carlos, Metepec, Estado de México. C. P. 52159, tel: 7223502850, e-mail: julieta_garay@yahoo.com. Mtra. Juana María de la Luz Esteban Valdez. Dirección: Paseo de los Sauces 174, Fraccionamiento Casa Blanca, Metepec, Estado de México. C. P. 52175, tel. 7222643584, e-mail: juanitapercastre@yahoo.com.mx. Dra. Gloria Margarita Gurrola Peña. Dirección: Casa 1b. 3ra. Cerrada de Tlalpan, col. San Jorge. Toluca, Estado de México. C. P. 50100, tel: 7223370363, e-mail: mgurrolaunid@hotmail.com. Dra. Patricia Balcázar Nava. Dirección: Abeto 78-A, fraccionamiento Ahuehuetes, Toluca, Estado de México. C. P. 50206, tel: 7223403460, e-mail: pbalcazarnava@hotmail.com
} 
De acuerdo con Oblitas (2006), el estrés implica un estado corporal o fisiológico de la activación o la tensión generalizada de lo que está ocurriendo, como respuesta ante ciertas situaciones o demandas ambientales. Lazarus (2000) menciona que a partir del análisis que realizó Hooke se pueden estudiar tres conceptos: carga, estrés y tensión. La carga se refiere a las fuerzas externas, como el peso; el estrés es el área de estructura sobre la que se aplica la carga y la tensión es la deformación de la estructura, producida por la carga y el estrés.

En palabras de Lazarus y Folkman (1986), el estrés psicológico es "una relación particular entre el individuo y el entorno que es evaluado por éste como amenazante o desbordante de sus recursos y que pone en peligro su bienestar".

Estas características han hecho que se les considere como acontecimientos con los que tropiezan las personas. Estos desencadenantes implican cambios en las rutinas de la vida cotidiana, rupturas con sus automatismos, lo que provoca nuevas condiciones y necesidades ante las cuales la persona tiene que valorar su forma de responder.

Dohrenwend y Dohrenwend (1981) y Harris, Brown y Harris (1989), mencionan que el hecho de conseguir o dejar un empleo, el graduarse, el nacimiento de los hermanos, la muerte de un padre, son sucesos demarcadores en el ciclo vital e implican cambios y transiciones. Estos hechos externos se relacionan con los cambios internos mucho más profundos. El sistema externo está dado por la participación en un determinado entorno social que provee sus normas y valores, los cuales se reflejan en el sistema interno por la significación que adquieren para cada uno.

Por otra parte, Oblitas y Becoña (2001) refieren que las personas suelen considerar al estrés como exigencias externas que el ambiente les plantea, como un cambio de trabajo o la enfermedad de algún familiar. Un suceso o acontecimiento vital es todo hecho o circunstancia en la vida de una persona capaz de alterar o modificar sus condiciones de salud psicofísica.

En términos globales puede afirmarse que todo acontecimiento vital es en sí una experiencia social, posee significación psicológica, ocurre en un momento o etapa determinados, es percibido con cierta intensidad por el sujeto y tiene una duración limitada en el tiempo.
Estudios realizados acerca de sucesos vitales estresantes y depresivos durante la adolescencia (Avison \& Turner, 1988; Compas, Slavin, Wagner \& Vannata; 1986), indican que el uso de escalas auto administrables que evalúan el impacto de situaciones de estrés brindan información útil acerca del efecto de factores crónicos.

Lazarus y Cohen (1977) introducen el concepto de afrontamiento como un conjunto de esfuerzos, conductas o pensamientos tendientes a manejar, tolerar, reducir o minimizar las demandas y conflictos, sean internos o externos. Asimismo, Lazarus y Folkman (1986) mencionan que el afrontamiento implica una serie de conductas que las personas emplean para cubrirse de un posible daño ante situaciones nuevas y desarrollan un modelo en el que los estresores se definen como transacciones o intercambios entre la persona y los diferentes contextos ambientales que se perciben como amenazantes. El individuo se conceptualiza como un agente activo en sus relaciones con el medio, en tanto que el afrontamiento se concibe como los cambios psicológicos de la persona en respuesta a las demandas transaccionales.

De acuerdo con Fleming, Baum y Singer (1984), el afrontamiento es una reacción al estrés, ya sea conductual o psicológica, dirigida a reducir sus cualidades abrasivas. Son aquellos aspectos que incluyen los intentos del individuo para resistir y superar al estresor. Por otra parte, el afrontamiento también se refiere a una gran variedad de reacciones (fisiológicas cognitivas, motoras) que controlan cualquier demanda sobre el individuo, ya sea en forma directa o por medio de interpretar anticipadamente sus consecuencias (Zegan, 1982).

\section{MÉTODO}

\section{Participantes}

Se trabajó con 84 pacientes diagnosticados con cáncer que acuden al Centro Oncológico del IsSEMYM; 21 son hombres y 63 mujeres, 44 de los cuales tienen estudios básicos, es decir, primaria o secundaria, y 40 de ellos de nivel superior, que va desde bachillerato, licenciatura o posgrado; las edades fluctúan entre los 20 a los 81 años, siendo el promedio de 63 años de los pacientes que tienen menos de un año acudiendo al Centro 
Oncológico. Veintiún pacientes llevan más de un año en tratamiento. El 79.8\% son católicos y el 53.6\% tienen seguro médico del Issemym.

\section{Instrumentos}

Para mediar los acontecimientos productores del estrés se empleó la escala elaborada por Caballo (1994), la cual mide el estrés según cada persona lo atribuye a una determinada situación; el instrumento consta de 52 ítems con intervalos de 1 a 10. La puntuación global del instrumento se obtiene sumando el total de los números marcados en cada uno de los reactivos, y se encuentra dividido en cinco factores, denominados: agresión física o ambiental, muerte o problemas de salud, problemas con la familia u otras personas, problemas con la pareja y algún cambio importante de la vida. La validación y confiabilidad fue realizada por Garay (2005), obteniendo el instrumento un alfa de Cronbach total de .960 y una varianza del 56\%.

Para mediar las estrategias de afrontamiento se empleó el instrumento elaborado por Carven, Scheier y Weintraub (1989) y adaptado en España por Crespo y Cruzado en 1997; en México se realizó la validación del Instrumento por Moysén (2011), y quedó conformado por siete factores denominados: planeación y afrontamiento activo, apoyo social, aceptación, apoyo en la religión, evasión, consumo de alcohol y drogas y humor; el alfa total del instrumento es de .915 y la varianza del $39 \%$.

\section{Procedimiento}

Se solicitó la colaboración de los pacientes previamente diagnosticados con cáncer y que asisten a consulta, así como su autorización para participar en la investigación, para lo cual se les pidió que firmaran una carta

Tabla 1. Frecuencia y porcentaje del nivel de estrés en pacientes con cáncer

\begin{tabular}{ccc}
\hline Nivel de Estrés & Frecuencia & Porcentaje \\
\hline Muy poco & 24 & 28.6 \\
\hline Medio & 47 & 56.0 \\
\hline Alto & 13 & 15.5 \\
\hline
\end{tabular}

de consentimiento; la aplicación fue individual, en una sola sesión.

\section{RESULTADOS}

De acuerdo con los resultados obtenidos del nivel de estrés de los pacientes con cáncer se observa que un porcentaje mayor (56\%) presenta un nivel de estrés medio, el $28.6 \%$ se encuentra con muy poco estrés y el $15.5 \%$ con estrés alto. (ver tabla 1 ).

Con respecto a los acontecimientos productores del estrés, en la tabla 2 se observa que los sucesos relacionados con la muerte o problemas de salud son los que presentan la media más alta $(\mathrm{M}=7.2262)$. Esta escala se relaciona con los acontecimientos que se encuentran viviendo los participantes de la investigación, ya que fueron diagnosticados con cáncer, y el solo hecho de conocer el diagnóstico o estar en tratamiento les produce un alto nivel de estrés. La escala que les sigue en cuanto a un nivel de estrés medio es la de agresión física o ambiental, la cual incluye eventos relacionados con ser objeto de violación, ser víctima der otros abusos sexuales, de agresión física, sufrir un desastre natural: terremoto, inundación.

Con respecto a las estrategias de afrontamiento se observa en la tabla 3 que la media más alta se encuentra en el factor de apoyo a la religión $(\mathrm{M}=3.1935)$; es decir, las personas que padecen de cáncer tienden a manejar el estrés rezando, con la convicción de que Dios les ayudará con su enfermedad. La estrategia que se encuentra en segundo lugar es la relacionada con planeación y afrontamiento activo.

Tabla 2. Medias y desviación estándar de las escalas de acontecimientos productores del estrés

\begin{tabular}{lcc}
\hline & M & DE \\
\hline Agresión física o ambiental & 6.297 & 2.731 \\
\hline Muerte o problemas de salud & 7.226 & 2.834 \\
\hline $\begin{array}{l}\text { Problemas con la familia u } \\
\text { otras personas }\end{array}$ & 4.859 & 2.524 \\
\hline $\begin{array}{l}\text { Problemas con la pareja } \\
\text { Algún cambio importante en }\end{array}$ & 5.575 & 2.748 \\
\hline la vida & 4.118 & 2.375 \\
\hline
\end{tabular}

Nota: Los valores van de 1 a 10 
Tabla 3. Medias y desviación estándar de las estrategias de afrontamiento

\begin{tabular}{lcl}
\hline & $\mathrm{M}$ & $\mathrm{DE}$ \\
\hline $\begin{array}{l}\text { Planeación y } \\
\text { afrontamiento activo }\end{array}$ & 2.462 & .693 \\
\hline Apoyo social & 2.427 & .680 \\
\hline Aceptación & 2.461 & .802 \\
\hline Apoyo en la religión & 3.194 & .701 \\
\hline $\begin{array}{l}\text { Evasión } \\
\text { Consumo de alcohol y } \\
\text { drogas }\end{array}$ & 1.927 & .520 \\
\hline \begin{tabular}{l} 
Humor \\
\hline
\end{tabular}
\end{tabular}

Nota: Los valores van de 1 a 4
De acuerdo con los resultados obtenidos en la tabla 4, de la comparación de los acontecimientos productores de estrés por sexo se observa que existe diferencia estadísticamente significativa en los sucesos que tienen que ver con algún cambio importante en la vida; en este factor las mujeres presentan una media mayor que los hombres $(\mathrm{M}=4.5040>2.9553, \mathrm{p}=.003)$, lo cual hace referencia a que las mujeres perciben que les ha cambiado más la vida a partir del suceso que les produce estrés.

De acuerdo con los resultados obtenidos de la comparación de las estrategias de afrontamiento por escolaridad, se observa que existe diferencia estadísticamente significativa en los factores de planeación y afrontamiento activo, apoyo social, y humor, en donde la media más alta se ubica en los pacientes con cáncer que tienen estudios superiores (ver tabla 5).

Tabla 4. Comparación de los acontecimientos productores de estrés por sexo

\begin{tabular}{|c|c|c|c|c|c|c|c|}
\hline & \multicolumn{2}{|c|}{ Hombres } & \multicolumn{2}{|c|}{ Mujeres } & \multirow{2}{*}{$\mathrm{t}$} & \multirow{2}{*}{ gl } & \multirow[b]{2}{*}{$p$} \\
\hline & $M$ & $\mathrm{DE}$ & $M$ & $\mathrm{DE}$ & & & \\
\hline Agresión física o ambiental & 5.435 & 2.715 & 6.585 & 2.697 & 1.689 & 82 & .095 \\
\hline Muerte o problemas de salud & 6.554 & 3.016 & 7.450 & 2.759 & 1.260 & 82 & .211 \\
\hline $\begin{array}{l}\text { Problemas con la familia u } \\
\text { otras personas }\end{array}$ & 4.032 & 2.372 & 5.135 & 2.530 & 1.757 & 82 & .083 \\
\hline Problemas con la pareja & 4.912 & 2.640 & 5.796 & 2.768 & 1.282 & 82 & .203 \\
\hline $\begin{array}{l}\text { Algún cambio importante en } \\
\text { la vida }\end{array}$ & 2.958 & 1.730 & 4.504 & 2.446 & 3.171 & 82 & .003 \\
\hline
\end{tabular}

Tabla 5. Comparación de las estrategias de afrontamiento por escolaridad

\begin{tabular}{|c|c|c|c|c|c|c|c|}
\hline & \multicolumn{2}{|c|}{ Básica } & \multicolumn{2}{|c|}{ Superior } & \multirow{2}{*}{$\mathrm{t}$} & \multirow{2}{*}{ gl } & \multirow{2}{*}{$p$} \\
\hline & $M$ & $\mathrm{DE}$ & $M$ & $\mathrm{DE}$ & & & \\
\hline $\begin{array}{l}\text { Planeación y afrontamiento } \\
\text { activo }\end{array}$ & 2.274 & .718 & 2.668 & .609 & 2.695 & 82 & .009 \\
\hline Apoyo social & 2.245 & .668 & 2.628 & .642 & 2.672 & 82 & .009 \\
\hline Aceptación & 2.335 & .812 & 2.600 & .778 & 1.522 & 82 & .132 \\
\hline Apoyo en la religión & 3.159 & .743 & 3.231 & .659 & .469 & 82 & .640 \\
\hline Evasión & 1.886 & .551 & 1.971 & .486 & .742 & 82 & .460 \\
\hline Consumo de alcohol y drogas & 1.212 & .367 & 1.233 & .415 & .249 & 82 & .804 \\
\hline Humor & 1.744 & .683 & 2.069 & .705 & 2.141 & 82 & .035 \\
\hline
\end{tabular}


Los resultados obtenidos en la tabla 6 muestran la comparación de los acontecimientos productores del estrés por edad; se puede observar una media más alta en el factor de problemas con la familia u otras personas en los pacientes de 20 a 40 años $(M=5.6146)$, lo cual quiere decir que entre menor edad se tenga, más estrés se experimenta por problemas relacionados con los miembros de su familia o los amigos.

Con respecto a la comparación de las estrategias de afrontamiento por edad, se observa que existe diferencia estadísticamente significativa en la estrategia de consumo de alcohol y drogas ( $\mathrm{p}=.002)$; de acuerdo con los resultados los pacientes con cáncer de 20 a 40 años tienden a manejar más el estrés a través de esta estrategia. (ver tabla 7).

Con respecto a los resultados obtenidos de la comparación de las estrategias de afrontamiento y el tiempo de tratamiento, se observa que existe diferencia estadísticamente significativa en el factor de aceptación ( $\mathrm{p}=.028)$; de acuerdo con las medias de los grupos el que lleva menos de un año con el tratamiento tiene la media más alta $(M=2.5714)$, lo que quiere decir que manejan más el estrés con aceptación que los que tienen más de un año de tratamiento (ver tabla 8).

Tabla 6. Comparación de los acontecimientos productores de estrés por edad

\begin{tabular}{|c|c|c|c|c|c|c|c|}
\hline & \multicolumn{2}{|c|}{20 a 40 años } & \multicolumn{2}{|c|}{ Más de 40 años } & \multirow{2}{*}{$\mathbf{t}$} & \multirow{2}{*}{ gl } & \multirow{2}{*}{$\mathrm{p}$} \\
\hline & M & $\mathrm{DE}$ & M & DE & & & \\
\hline Agresión física o ambiental & 6.552 & 2.453 & 6.141 & 2.901 & .667 & 82 & .506 \\
\hline Muerte o problemas de salud & 7.797 & 2.632 & 6.875 & 2.920 & 1.458 & 82 & .149 \\
\hline $\begin{array}{l}\text { Problemas con la familia u otras } \\
\text { personas }\end{array}$ & 5.615 & 2.550 & 4.394 & 2.415 & 2.202 & 82 & .030 \\
\hline Problemas con la pareja & 6.281 & 2.450 & 5.140 & 2.852 & 1.876 & 82 & .064 \\
\hline Algún cambio importante en la vida & 4.590 & 2.382 & 3.827 & 2.347 & 1.439 & 82 & .154 \\
\hline
\end{tabular}

Tabla 7. Comparación de las estrategias de afrontamiento por edad

\begin{tabular}{|c|c|c|c|c|c|c|c|}
\hline & \multicolumn{2}{|c|}{20 a 40 años } & \multicolumn{2}{|c|}{ Más de 40 años } & \multirow{2}{*}{$\mathrm{t}$} & \multirow{2}{*}{ gl } & \multirow{2}{*}{$\mathrm{p}$} \\
\hline & $M$ & $\mathrm{DE}$ & $M$ & $\mathrm{DE}$ & & & \\
\hline Planeación y afrontamiento activo & 2.299 & .621 & 2.562 & .722 & 1.706 & 82 & .092 \\
\hline Apoyo social & 2.434 & .720 & 2.423 & .660 & .071 & 82 & .943 \\
\hline Aceptación & 2.391 & .823 & 2.505 & .794 & .631 & 82 & .530 \\
\hline Apoyo en la religión & 3.008 & .761 & 3.308 & .643 & 1.935 & 82 & .056 \\
\hline Evasión & 2.037 & .453 & 1.859 & .550 & 1.533 & 82 & .129 \\
\hline Consumo de alcohol y drogas & 1.385 & .494 & 1.122 & .264 & 3.183 & 82 & .002 \\
\hline Humor & 1.945 & .698 & 1.870 & .720 & .470 & 82 & .640 \\
\hline
\end{tabular}


Tabla 8. Comparación de las estrategias de afrontamiento por tiempo de tratamiento

\begin{tabular}{|c|c|c|c|c|c|c|c|}
\hline & \multicolumn{2}{|c|}{ Menos de un año } & \multicolumn{2}{|c|}{ Más de un año } & \multirow[t]{2}{*}{$\mathrm{t}$} & \multirow[t]{2}{*}{ gl } & \multirow[t]{2}{*}{$\mathrm{p}$} \\
\hline & $M$ & $\mathrm{DE}$ & $M$ & $\mathrm{DE}$ & & & \\
\hline Planeación y afrontamiento activo & 2.493 & .693 & 2.367 & .702 & .718 & 82 & .475 \\
\hline Apoyo social & 2.471 & .684 & 2.296 & .666 & 1.020 & 82 & .311 \\
\hline Aceptación & 2.571 & .806 & 2.131 & .705 & 2.230 & 82 & .028 \\
\hline Apoyo en la religión & 3.210 & .702 & 3.143 & .714 & .380 & 82 & .705 \\
\hline Evasión & 1.955 & .538 & 1.841 & .461 & .868 & 82 & .388 \\
\hline Consumo de alcohol y drogas & 1.222 & .397 & 1.222 & .370 & .000 & 82 & 1.000 \\
\hline Humor & 1.901 & .704 & 1.893 & .740 & .044 & 82 & .965 \\
\hline
\end{tabular}

Tabla 9. Correlación entre los acontecimientos productores de estrés y estrategias de afrontamiento en pacientes oncológicos

\begin{tabular}{|c|c|c|c|c|c|c|c|}
\hline & $\begin{array}{l}\text { Agresión } \\
\text { física o } \\
\text { ambiental }\end{array}$ & $\begin{array}{l}\text { Muerte o } \\
\text { problemas } \\
\text { de salud }\end{array}$ & $\begin{array}{c}\text { Problemas con } \\
\text { la familia u } \\
\text { otras personas }\end{array}$ & $\begin{array}{c}\text { Problemas } \\
\text { con la } \\
\text { pareja }\end{array}$ & $\begin{array}{c}\text { Algún cambio } \\
\text { importante en } \\
\text { la vida }\end{array}$ & $M$ & $\mathrm{DE}$ \\
\hline $\begin{array}{l}\text { Planeación y } \\
\text { afrontamiento activo }\end{array}$ & $.227^{*}$ & $.219 *$ & .146 & .138 & .149 & 2.462 & .693 \\
\hline Apoyo social & .202 & $.238 *$ & .093 & .131 & .108 & 2.427 & .680 \\
\hline Aceptación & $.232 *$ & $.249 *$ & .084 & .123 & .023 & 2.461 & .802 \\
\hline Apoyo en la religión & .183 & .156 & .134 & .070 & .075 & 3.194 & .701 \\
\hline Evasión & $.281 * *$ & $.280 * *$ & $.238 *$ & $.269 *$ & .208 & 1.927 & .520 \\
\hline $\begin{array}{l}\text { Consumo de alcohol } \\
\text { o drogas }\end{array}$ & .053 & .129 & $.228^{*}$ & .207 & $.254^{*}$ & 1.222 & .388 \\
\hline Humor & -.051 & .021 & -.098 & .000 & -.017 & 1.899 & .708 \\
\hline$M$ & 6.297 & 7.226 & 4.859 & 5.575 & 4.118 & & \\
\hline $\mathrm{DE}$ & 2.731 & 2.834 & 2.524 & 2.748 & 2.375 & & \\
\hline
\end{tabular}

Nota: * $p \leq .05$

${ }^{* *} \mathrm{p} \leq .01$

De acuerdo con los resultados obtenidos de la correlación de los acontecimientos productores del estrés y las estrategias de afrontamiento en pacientes oncológicos se observan 11 correlaciones positivas; los acontecimientos de agresión física o ambiental correlacionan positivamente con la estrategia de afrontamiento de planeación y afrontamiento activo, aceptación y evasión. Los acontecimientos de muerte o problemas de salud correlacionan positivamente con planeación y afrontamiento activo, apoyo social, aceptación y evasión. Por otra parte, los sucesos del área de problemas de la familia u otras personas correlacionan de forma positiva con evasión y consumo de alcohol y drogas. Mientras que los problemas con la pareja correlacionan con evasión, al igual que los acontecimientos de algún cambio importante en la vida (ver tabla 9). 


\section{DISCUSIÓN}

De acuerdo con los resultados obtenidos, el 56\% de los pacientes con cáncer que acuden al Centro Oncológico presentan un nivel de estrés medio; el $28.6 \%$ se encuentra con muy poco estrés y el $15.5 \%$ con estrés alto. Cabe hacer mención que según lo reportado por Larse y Buss (2005), el estrés es un sentimiento subjetivo producido por los sucesos que son incontrolables o amenazadores.

Según estudios previos, realizados por Lazarus y Folkman (1984) y Caballo y Simon (2006), el estrés se puede medir a través de los acontecimientos vitales. Asimismo, los autores mencionan que estos sucesos juegan un papel en el desarrollo de enfermedades, indicando que la amenaza o lo desagradable de la situación se encuentra implicada en la salud de las personas.

Los acontecimientos vitales se han definido como eventos sociales que requieren de algún tipo de cambio con respecto al habitual ajuste del individuo. El potencial estresante está en función de la cantidad de cambio que conlleva. Con respecto a los acontecimientos productores del estrés se observa que los sucesos relacionados con la muerte o problemas de salud son los que presentan la media más alta $(M=7.2262)$; esta escala se relaciona con los acontecimientos que se encuentran viviendo los participantes de la investigación, ya que fueron diagnosticados con cáncer, y el sólo hecho de conocer el diagnóstico o de estar en tratamiento les produce un alto nivel de estrés. La escala que les sigue es la de agresión física o ambiental, la cual incluye eventos relacionados con ser objeto de violación, ser víctima de otros abusos sexuales, ser de agresión física, sufrir un desastre natural: terremoto, inundación.

Con respecto a las estrategias de afrontamiento se observa que la media más alta se encuentra en el factor de apoyo a la religión ( $M=3.1935)$, es decir, las personas que padecen de cáncer tienden a manejar el estrés a través de rezar, confiando en Dios para que les ayude con su enfermedad. De acuerdo con Taylor (2007) la religión puede promover un sentimiento de bienestar psicológico. Las personas con una fe religiosa fuerte reportan tener una mayor satisfacción en la vida, mayor felicidad personal y menos consecuencias negativas en eventos traumáticos.

La estrategia que se encuentra en segundo lugar es la relacionada con planeación y afrontamiento activo.
De acuerdo con los resultados obtenidos de la comparación de los acontecimientos productores de estrés y el sexo se observa que existe diferencia estadísticamente significativa en los sucesos que tienen que ver con algún cambio importante en la vida en el cual las mujeres presentan una media mayor que los hombres $(\mathrm{M}=4.5040>2.9553, \mathrm{p}=.003)$, lo cual hace referencia a que las mujeres perciben que les ha cambiado más la vida a partir del suceso que les produce estrés.

De acuerdo con los resultados obtenidos de la comparación de las estrategias de afrontamiento por escolaridad, se observa que existe diferencia estadísticamente significativa en los factores de planeación y afrontamiento activo, apoyo social, y humor, en donde la media más alta se ubica en los pacientes con cáncer que tienen estudios superiores.

Con respecto a la comparación de las estrategias de afrontamiento por edad, se observa que existe diferencia estadísticamente significativa en la estrategia de consumo de alcohol y drogas ( $\mathrm{p}=.002$ ); de acuerdo con los resultados, los pacientes con cáncer de 20 a 40 años tienden a manejar más el estrés a través de esta estrategia.

De acuerdo con los resultados obtenidos se observaron 11 correlaciones positivas entre los acontecimientos productores del estrés y las estrategias de afrontamiento en pacientes oncológicos. Específicamente, los acontecimientos de agresión física o ambiental correlacionan positivamente con la estrategia de afrontamiento de planeación y afrontamiento activo, aceptación y evasión. Los acontecimientos de muerte o problemas de salud correlacionan positivamente con planeación y afrontamiento activo, apoyo social, aceptación y evasión. Los sucesos del área de problemas de la familia u otras personas correlacionan de forma positiva con evasión y consumo de alcohol y drogas. Los problemas con la pareja correlacionan con evasión, al igual que los acontecimientos de algún cambio importante en la vida. 


\section{REFERENCIAS}

Avison, W. \& Turner, J. (1988). Stressful Life Events and Depressive Symptoms: Desaggregrating the Effects of Stressor and Chronic Strain. Journal of Health and Social Behavior, 29(2), 53-564.

Caballo, V. E. (1994). The scale of stressful life events (SSLE). España: Universidad de Granada.

Caballo, V. E. \& Simón, M. A. (2006). Acontecimientos vitales estresantes y trastornos psicopatológicos. En G. L. A. Oblitas. Psicología de la Salud. México: Plaza y Valdés.

Carver, Ch. S., Scheier, M. F. \& Weintraub, J. K. (1989). Cuestionario Cope, en T. Gutiérrez, R. Raich, D. Sánchez \& J. Deus (2002). Instrumentos de evaluación en psicología de la salud. Barcelona: Alianza Editorial.

Compas, B., Slavin, L., Wagner, B. \& Vannata, K. (1986). Relationship of Life Events and Social Support with Psychological Disfunctions Among Adolescents. Journal of Youth and Adolescence, 15(3), 205-221.

Crespo, M. L. \& Cruzado, J. A. (1997). La evaluación del afrontamiento: adaptación del cuestionario cope con una muestra de estudiantes universitarios. Análisis y modificación de la conducta, 23(92), 797-830.

Dohrenwend, B. S. \& Dohrenwend, B. P. (1981). Stressful Life Events. Their nature and their Context, Nueva Jersey: Rutgers University Press.

Fleming, R., Baum, A. \& Singer, J. E. (1984). Toward and integrative approach to the study of stress. Journal of personality and social psychology, 46(1), 939-949.

Garay, L. J. C. (2005). Niveles de depresión, autoestima y estrés en mujeres que tienen un trabajo remunerado y mujeres que no tienen un trabajo remunerado (amas de casa). (Tesis de doctorado). Universidad Iberoamericana: México.

Harris, T., Brown, G. \& Bifulco, A. (1990). Loss of parent in childhood and adult psychiatric disorder: A tentative overall model. Development and psychopatholohy, 2(1), 311-328.

Larse, R. L. \& Buss, D. M. (2005). Psicología de la personalidad. México: McGraw Hill.

Lazarus, R. S. \& Cohen, J. B. (1977). Envionmental stress. Human behavior and the envionment: current theory and research. Nueva York: Plenum.

Lazarus, R. S. \& Folkman, S. (1984). Stress, Appraisal and Doping. Nueva York: Springer Publishing Company. (Trad. Martinez Roca, 1986).

Lazarus, R. \& Folkman, S. (1986). Estrés y procesos cognitivos. Barcelona: Martínez Roca.

Lazarus, R. S. (2000). Estrés y emoción. Manejo e implicaciones en nuestra salud. España: Desclée de Brouwer.

Moysén, C. A. (2011). Modelo de consumo de alcohol en estudiantes universitarios a través de los sucesos de vida y estrategias de afrontamiento. (Tesis de doctorado). Universidad Iberoamericana: México.

Oblitas, L. (2006). Psicología de la salud y calidad de vida. México: Thompson.

Oblitas, L. \& Becoña, E. (2001). Psicología de la Salud. México: Plaza y Valdés Editores.

Taylor, S. (2007). Psicología de la salud. México: McGraw Hill.

Zegan, L. S. (1982). Stress and development of somatic disorder. Handbook of Stress. Nueva York: Godberger \& Breznitz, Free Press 